\title{
CtBP2 is associated with angiogenesis and regulates the apoptosis of prostate cancer cells
}

\author{
QIANG XUAN ${ }^{1 *}$, XIAOGE ZHONG ${ }^{2 *}$, WEIDONG LI $^{3}$, ZENGNAN MO ${ }^{2}$, \\ YUANJIE $\mathrm{HUANG}^{3}$ and YANLING $\mathrm{HU}^{2-4}$ \\ ${ }^{1}$ Department of Urology, Anhui Provincial Hospital Affiliated Anhui Medical University, Hefei, Anhui 230022; \\ ${ }^{2}$ Center for Genomic and Personalized Medicine, Guangxi Medical University, Nanning, Guangxi 530021; \\ ${ }^{3}$ Life Sciences Institute, Guangxi Medical University, Nanning, Guangxi 530021; ${ }^{4}$ Guangxi Colleges and \\ Universities Key Laboratory of Biological Molecular Medicine Research, Guangxi Medical University, \\ Nanning, Guangxi 530021, P.R. China
}

Received October 28, 2016; Accepted May 8, 2017

DOI: 10.3892/or.2017.5763

\begin{abstract}
Angiogenesis is associated with prostate cancer (PCa) development and progression. Aberrant expression of C-terminal binding protein (CtBP) 2 has been observed in $\mathrm{PCa}$, but whether its change in expression plays a significant role in angiogenesis has not been completely characterized. we attempted to integrate and analyze the genome-wide association study (GWAS) of follicle stimulating hormone receptor (FSHR) and CtBP2, the Cancer Genome Atlas (TCGA) data and CtBP2 binding data in CistromeMap (18) to explore the mechanism of CtBP2 in PCa, and performed pathway enrichment analysis. We revealed that the top 6 pathways were closely related with angiogenesis. We used siRNA and overexpression plasmids to silence and overexpress CtBP2 expression. Altered expression of CtBP2 affected the expression of VEGFA, FSHR, FHL2 and SMAD3 which are closely related with angiogenesis. In addition, silencing of $\mathrm{CtBP} 2$ markedly increased the apoptosis of $\mathrm{PCa}$ cells in vitro, and decreased the expression of IL-8, AT2R, CCND1 and MMP9 which are associated with cancer progression. These results highlight the association between CtBP2 and angiogenesis in $\mathrm{PCa}$ and indicate that $\mathrm{CtBP} 2$ may be a potential therapeutic target for $\mathrm{PCa}$.
\end{abstract}

Correspondence to: Professor Yanling Hu, Center for Genomic and Personalized Medicine, Guangxi Medical University, Nanning, Guangxi 530021, P.R. China

E-mail: huyanling@gxmu.edu.cn

Professor Yuanjie Huang, Life Sciences Institute, Guangxi Medical University, Nanning, Guangxi 530021, P.R. China

E-mail: renee927@126.com

*Contributed equally

Key words: $\mathrm{CtBP} 2$, angiogenesis, apoptosis, prostate cancer

\section{Introduction}

Prostate cancer $(\mathrm{PCa})$ is one of the most common cancers of the male reproductive system and the second leading cause of cancer-related death among men worldwide. In 2015, 41,210 cancer deaths were estimated among males, where 6,801 were from PCa (1). At present, for patients diagnosed with clinically localized cancer, effective treatments for $\mathrm{PCa}$ include radical prostatectomy, radiotherapy or androgen-deprivation therapy. However, approximately $15 \%$ of patients present with local recurrence or develop metastatic tumors (2). Additionally, some patients develop castration-resistant prostate cancer (CRPC), for which treatment is limited and the death rate is high $(3,4)$. Therefore, it is important to clarify the mechanism of tumor development and progression to optimize therapeutic strategies for PCa.

The C-terminal binding proteins (CtBPs) were initially identified as binding partners of the E1A protein (5). The $\mathrm{CtBP}$ family members $\mathrm{CtBP} 1$ and $\mathrm{CtBP} 2$ are highly homologous transcriptional corepressors, which are conserved in both vertebrates and invertebrates (6). Recent studies have observed aberrant expression of CtBPs in many human malignancies, such as ovarian cancer, melanoma, breast cancer and esophageal squamous cell carcinoma (7-10). Growing evidence shows that $\mathrm{CtBP} 2$ is aberrantly upregulated in $\mathrm{PCa}$ and its expression is associated with cancer development and progression (11-13).

Angiogenesis is an essential process in tumor development, and is an important focus of cancer research and therapy and may be a target of cancer therapy as the survival and proliferation of cancer depend on angiogenesis (14). Among the many molecular markers associated with tumor angiogenesis, the Tel gene has been shown to play an evolutionarily conserved role in angiogenesis. Tel is indispensable for the sprouting of human endothelial cells and for normal development of the Danio rerio blood circulatory system. Tel orchestrates endothelial sprouting by binding to the generic co-repressor, CtBP, and forms a Tel-CtBP complex, which temporally restricts a vascular endothelial growth factor (VEGF)-mediated pulse of D1l4 expression and thereby directly links VEGF receptor intracellular signaling and intercellular Notch-D114 
signaling (15). Thus, we propose that $\mathrm{CtBP} 2$ may play an important role in angiogenesis in $\mathrm{PCa}$.

Follicle stimulating hormone receptor (FSHR) is thought to play a critical role in reproductive physiology in the ovary and spermatogenesis by promoting follicular growth and estrogen synthesis. Stilley et al (16) revealed that FSH is as efficacious as VEGF in promoting angiogenic processes and stimulating angiogenesis directly. Yang et al (17) demonstrated that FSHR is a highly selective tumor vasculature marker in both primary and metastatic tumors. In this study, we attempted to integrate and analyze the genome-wide association study (GWAS) of FSHR and CtBP2, the Cancer Genome Atlas (TCGA) data and $\mathrm{CtBP} 2$ binding data in CistromeMap (18) to explore the mechanism of $\mathrm{CtBP} 2$ in $\mathrm{PCa}$.

\section{Materials and methods}

Bioinformatic analysis. First, mRNA expression of CtBP2 and FSHR were extracted from lymphoblastoid cell lines of 210 HapMap unrelated individuals at the NCBI's Gene Expression Omnibus website (http://www.ncbi.nlm.nih. gov/geo/); series number GSE6536 (19). In order to find a relationship between $\mathrm{CtBP} 2$ and FSHR expression and the corresponding Genome-wide genotype, the phase III genotype of these 210 unrelated individuals was downloaded from the HapMap website. We used Plink software to carry out the association analysis between CtBP2 and FSHR expression and the corresponding Genome-wide genotype on Chinese (CHB) and Japanese (JPT) populations. The annotation of these SNPs was performed in SNPfunc (http://snpinfo.niehs.nih.gov/snpinfo/ snpfunc.htm) (20). CtBP2 binding data in LNCaP cell lines was downloaded from CistromeMap database (18). All clinical and mRNA expression levels (level 3 data, RNA-seq version 2) of PCa patients (PRAD) were downloaded from TCGA data portal (https://tcga-data.nci.nih.gov/docs/ publications/tcga/) until August 29, 2015. The patients who suffered from other malignancies or received neoadjuvant therapy were removed.

Cell culture. The prostate carcinoma cell lines LNCaP, DU145 and PC3 were obtained from the American Type Culture Collection (ATCC nos. HTB-81 ${ }^{\mathrm{TM}}$, CRL-1435 ${ }^{\mathrm{TM}}$, CRL-1740 $\left.{ }^{\mathrm{TM}}\right)$. All cell lines were cultured in T-75 flasks to $80 \%$ confluence at $37^{\circ} \mathrm{C}$ in a humidified incubator containing $5 \% \mathrm{CO}_{2}$ (Thermo Fisher Scientific). LNCaP cells were grown in RPMI-1640 medium, DU145 and PC3 cells in Dullbecco's modified Eagle's medium (DMEM/F12; Hyclone, Logan, UT, USA) both supplemented with $10 \%$ fetal bovine serum (FBS; Thermo Fisher Scientific, Waltham, MA, USA) and 1\% penicillin/streptomycin $(100 \mathrm{U} / \mathrm{ml}$ penicillin and $100 \mu \mathrm{g} / \mathrm{ml}$ streptomycin; Hyclone).

RNA interference assay. LNCaP, DU145 and PC3 cells were transfected with various siRNAs targting $\mathrm{CtBP} 2$ (catalog nos.: CtBP2-Homo-451, CtBP2-Homo-778, CtBP2-Homo-1021), and negative siRNA with a random sequence was used as a control (Suzhou Genepharma Co., Ltd., Suzhou, China). The cells were seeded into 6 -well plates at a density of $5 \times 10^{5}$ cells/ well, and cultured at $37^{\circ} \mathrm{C}$ with $5 \% \mathrm{CO}_{2}$ until the cells reached $80 \%$ confluency. Lipofectamine 2000 reagent (Thermo Fisher Scientific, Inc.) was used for the transfections according to the
Table I. Sequences of CtBP2-siRNA and negative siRNA.

SiRNA Sequence, 5'-3'

$\begin{array}{ll}\text { CtBP2-siRNA-451 } & \\ \text { Sense } & \text { GACAGCGAUUGGACAGAAUTT } \\ \text { Antisense } & \text { AUUCUGUCCAAUCGCUGUCTT } \\ \text { CtBP2-siRNA-778 } & \\ \text { Sense } & \text { GAAUUGCCGUGUGCAACAUTT } \\ \text { Antisense } & \text { AUGUUGCACACGGCAAUUCTT } \\ \text { CtBP2-siRNA-1021 } & \\ \text { Sense } & \text { CCUUUGGAUUCAGCGUCAUTT } \\ \text { Antisense } & \text { AUGACGCUGAAUCCAAAGGTT } \\ \text { Negative siRNA } & \\ \text { Sense } & \\ \text { Antisense } & \text { UUCUCCGAACGUGUCACGUTT } \\ \end{array}$

CtBP2, C-terminal binding protein 2; siRNA, small interfering RNA.

Table II. Genes and their primer sequences from qPCR.

\begin{tabular}{ll}
\hline Genes & \multicolumn{1}{c}{ Primers } \\
\hline FtBP2 & R: ACTGTGGCCTTCTGTGACGC \\
FSHR & F: AGCCTCTGGACCAGTCATTC \\
& R: ACAGCAATGGCTGGGATAGG \\
VEGFA & F: TCAGCGCAGCTACTGCCATC \\
& R: ACACTCCAGGCCCTCGTCATTG \\
FHL2 & F: CAGACTGCTATTCCAACGAG \\
RMAD3 & F: TAGCAGATGAAGCAGGTCTC \\
R: ATGGAATGGCTGTAGTCGTC \\
F: TTGGCAGCCTTCCTGATTCC \\
R: ATTTCTGTGTTGGCGCAGTG \\
FT: ATTGCTTCAGCCAGCGTCAG \\
R: TAGCTGGCAAACTGGCCAAG \\
F: ACCACGGCCAACTACGACAC \\
R: GTGCAGGCGGAGTAGGATTG \\
FCND1 & R: ATGGAACACCAGCTCCTGTG \\
& RAGATGTCCACGTCCCGCA \\
\hline
\end{tabular}

F, forward; R, reverse; VEGF, vascular endothelial growth factor; $\mathrm{CtBP} 2, \mathrm{C}$-terminal binding protein 2; FSHR, FSH receptor.

manufacturer's instructions. The siRNA sequences are shown in Table I.

CtBP2 overexpression. LNCaP, DU145 and PC3 cells were cultured in 6-well plates and transiently transfected with $2 \mu \mathrm{g}$ of cDNA coding CtBP2, and the vector pcDNA3.1 was cloned into the cells. Lipofectamine 2000 reagent was used for transfection according to the manufacturer's protocol. The DNA 
A

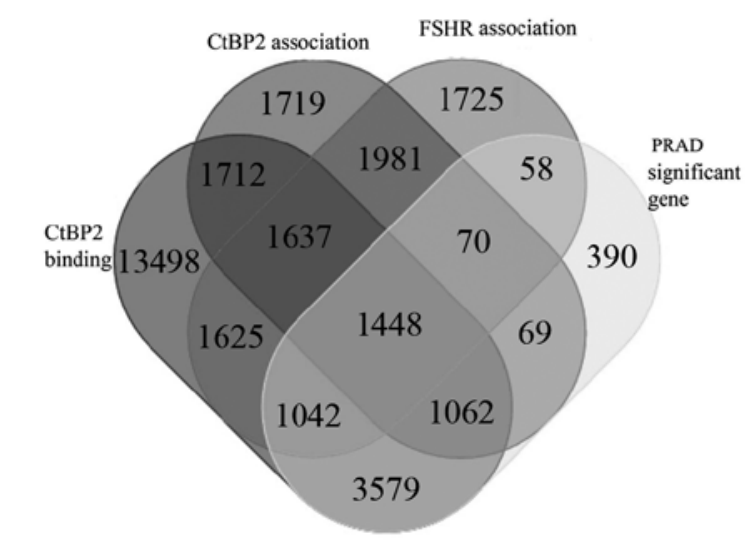

B

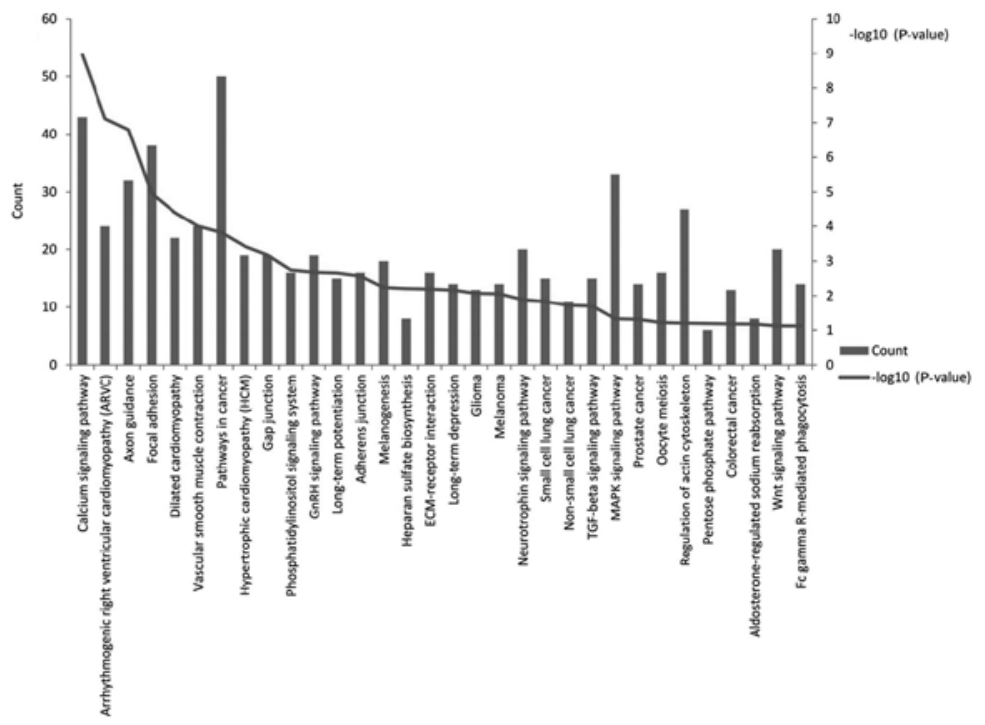

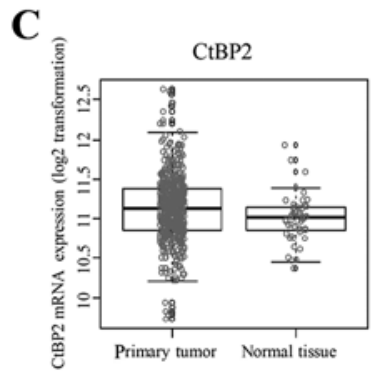
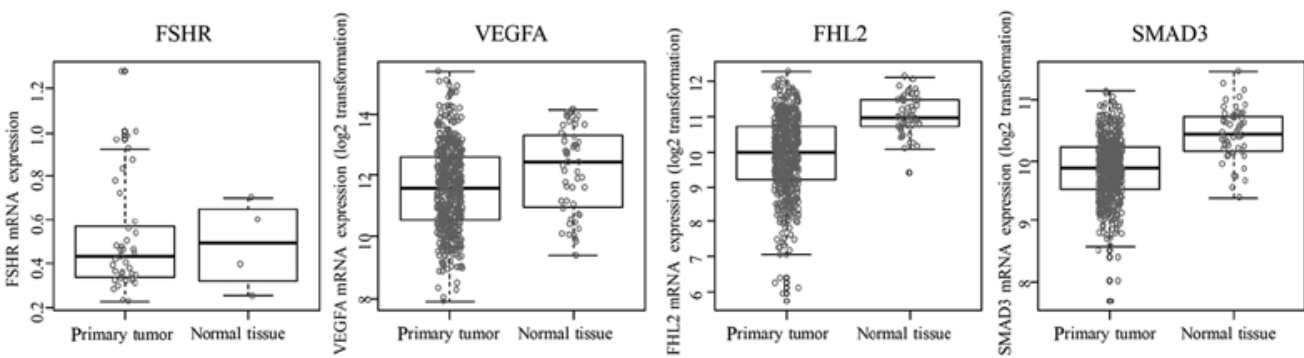

Figure 1. CtBP2 function mining by bioinformatic analysis. (A) Custom Venn/Euler diagrams for 4 datasets. Two datasets were the genes associated between CtBP2 or FSHR expression and HapMap genome-wide genotype, one dataset was the genes binding with CtBP2 in CistromeMap database (21), and one was the significant difference in mRNA expression between prostate cancer tissue and adjacent tissue from TCGA (PRAD). (B) Thirty-two pathways enriched in DAVID. (C) CtBP2, FSHR, VEGFA, FHL2 and SMAD3 expression between prostate cancer and adjacent tissues from TCGA. CtBP, C-terminal binding protein; FSHR, follicle stimulating hormone receptor; VEGF, vascular endothelial growth factor; FHL2, four and a half LIM domains 2; SMAD3, SMAD family member 3.

was diluted in OptiMEM (Invitrogen, Life Technologies Co., Carlsbad, CA, USA) combined with Lipofectamine 2000, and incubated for $20 \mathrm{~min}$ at room temperature. After incubation, the complex was added to the culture medium of each cell line, and cultured at $37^{\circ} \mathrm{C}$ with $5 \% \mathrm{CO}_{2}$.

$R T-P C R$. The expression of CtBP2 was analyzed after transfection. The primers for CtBP2 were as follows: 5'-ACTGTG GCCTTCTGTGACGC-3' forward and 5'-CTGGTGAGGGT GATGGTGTG-3' reverse. Glyceraldehyde-3-phosphate dehydrogenase (GAPDH) was used as a normalization control and was also amplified, and the primers were: 5'-TCATGAAGTGT GACGTGGACATC-3' forward and 5'-CAGGAGGAGCAAT GATCTTGATCT-3' reverse. The PCR reaction conditions were as follows: denaturation at $98^{\circ} \mathrm{C}$ for $3 \mathrm{~min} ; 32$ cycles of denaturation at $98^{\circ} \mathrm{C}$ for $10 \mathrm{sec}$; annealing at $60^{\circ} \mathrm{C}$ for $30 \mathrm{sec}$; and extension at $72^{\circ} \mathrm{C}$ for $1 \mathrm{~min}$. The final extension was performed at $72^{\circ} \mathrm{C}$ for $5 \mathrm{~min}$.

The real-time PCR reaction was performed in biological triplicates using the Applied Biosystems StepOne Plus real-time PCR system (Applied Biosystems, USA). Relative expression values were calculated according to the $2^{-\Delta \Delta \mathrm{Ct}}$ method. $2^{-\Delta \Delta \mathrm{Ct}}$ represents the times ratio of target gene in the experimental group and the control group, and the formula was as follows: $\Delta \Delta \mathrm{Ct}=\Delta \mathrm{Ct}_{\text {experimental group }}-\Delta \mathrm{Ct}_{\text {control group }}$, where $\Delta \mathrm{Ct}=\mathrm{Ct}_{\text {target gene }}-\mathrm{Ct}_{\mathrm{GAPDH}}$.

Western blot analysis. The protein levels of CtBP2 after transfection with CtBP2-siRNA or pcDNA3.1 and the negative control were determined using the $\mathrm{Wes}^{\mathrm{TM}}$ (ProteinSimple, San Jose, CA, USA) system. Wes 12-230 kDa Master kit with split buffer (ProteinSimple) was selected for western analysis. Simple western analysis was carried out at room temperature under instrument default settings. The digital images were analyzed with Compass software (ProteinSimple) on Wes.

Flow cytometry. LNCaP, DU145 and PC 3 cells were collected $48 \mathrm{~h}$ after transfection, and cell density was adjusted to $1 \times 10^{6} \%$ $\mathrm{ml}$. Cell suspension $(0.5 \mathrm{ml})$ was placed in a centrifuge tube, and $1.25 \mu \mathrm{l}$ of Annexin V-FITC (Nanjing KeyGEN Biotech Development Co., Ltd.) was added to the centrifuge tube. The whole system was carried out in a dark room at room temperature for $15 \mathrm{~min}$, and centrifuged at $1,000 \mathrm{x}$ g for $5 \mathrm{~min}$. After the supernatant was discarded, the cells were gently treated with $0.5 \mathrm{ml}$ of cold-binding buffer to re-suspend. Ten microliters of propidium iodide (PI) was used and flow cytometry 

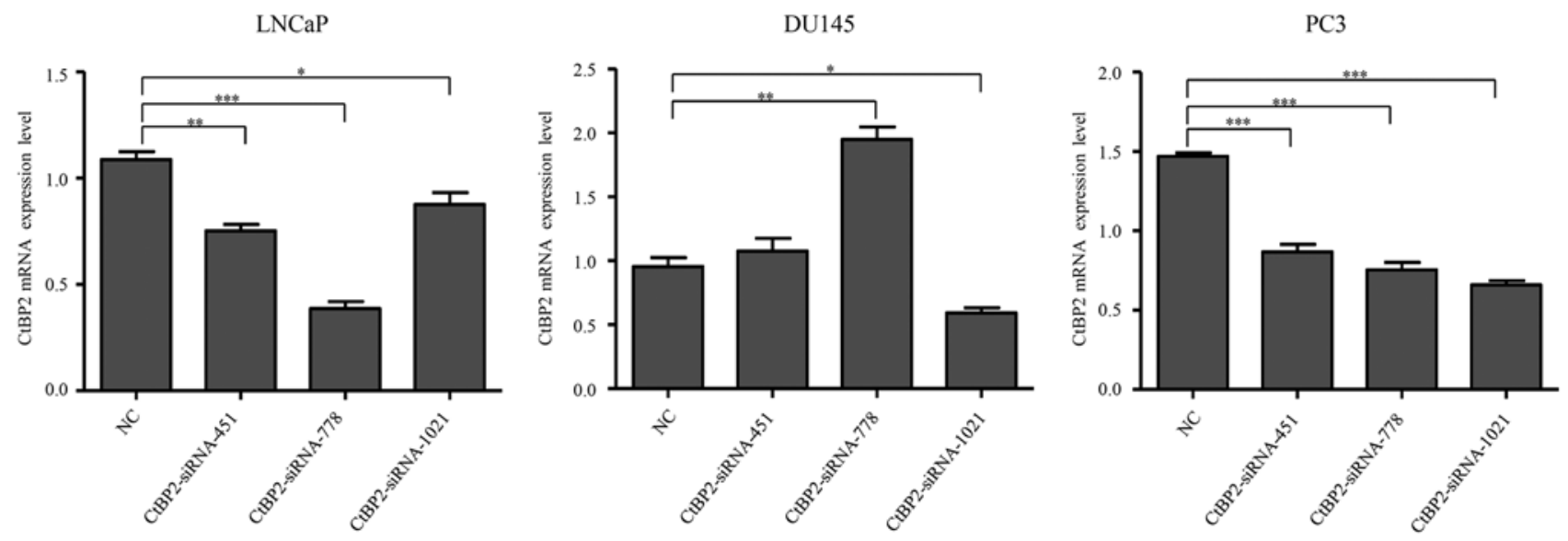

Figure 2. Knockdown of CtBP2 using siRNA in the PCa cell line. Reverse transcription-polymerase chain reaction of RNA extracted from LNCaP, DU145 and PC3 cells transfected with CtBP2-siRNA-451, CtBP2-siRNA-778, CtBP2-siRNA-1021 and negative siRNA. The data are expressed as mean \pm SEM. "P $<0.05$, ${ }^{* *} \mathrm{P}<0.01,{ }^{* * *} \mathrm{P}<0.001$. NC group transfected with negative siRNA. CtBP, C-terminal binding protein, PCa, prostate cancer.

(BD Biosciences, Franklin Lakes, NJ, USA) was immediately used for detection analysis.

Statistical analysis. Statistical analysis comparisons between groups were evaluated by using independent-samples t-test in SPSS 22.0 statistical software (SPSS Inc., Chicago, IL, USA). $\mathrm{P}<0.05$ was indicative of statistical significance. All values are expressed as mean \pm SEM.

\section{Results}

$C t B P 2$ function mining by bioinformatic analysis. We used an online tool (http://bioinformatics.psb.ugent.be/webtools/ Venn/) to generate custom Venn/Euler diagrams for 4 datasets, including the genes of two datasets associated between CtBP2 and FSHR expression and HapMap genome-wide genotype, one dataset of binding with CtBP2 in CistromeMap database (18), and one for the significant difference of mRNA expression between PCa and adjacent tissues from TCGA. Overlapping genes $(1,448)$ were found in these 4 datasets (Fig. 1A). Pathway enrichment was performed using DAVID (https://david.ncifcrf. gov/) (21), and 32 pathways were found to be enriched (Fig. 1B). Among these enriched pathways, the top 6 pathways were calcium signaling pathway, arrthythmogenic right ventricular cardiomyopathy (ARVC), axon guidance, focal adhesion and vascular smooth muscle contraction (VSMC) pathway. We further investigated 5 genes (CtBP2, FSHR, VEGFA, FHL2 and SMAD3) in the 1,448 overlapping genes which were closely correlated with angiogenesis (15-17,22-24). The results showed that there were significant differences between the expression of these 5 genes in the TCGA between PCa and normal adjacent tissues (Fig. 1C).

Knockdown of the CtBP2 gene using siRNAs in the PCa cell lines. In order to ascertain the potential function of CtBP2 in $\mathrm{PCa}$, we uses siRNAs to interfere with endogenous $\mathrm{CtBP} 2$ gene expression (gene ID, 1488). Overall, 3 siRNAs were transfected into LNCaP, DU145 and PC-3 cells, respectively, with transfection of the negative siRNA as control. Twenty-four hours after transfection of the siRNAs, CtBP2 mRNA expression was detected by RT-PCR. The results showed that, compared to the NC group, CtBP2-siRNA-451, CtBP2-siRNA-778 and CtBP2-siRNA-1021 significantly decreased CtBP2 expression in the LNCaP and PC-3 cells, while CtBP2-siRNA-451 and CtBP2-siRNA-778 increased CtBP2 expression in the DU145 cell lines, and only CtBP2-siRNA-1021 decreased CtBP2 in all cell lines. Therefore, CtBP2-siRNA-1021 was selected for the subsequent experiments (Fig. 2).

CtBP2 expression in LNCaP, DU145 and PC-3 cells after knockdown and overexpression. CtBP2-siRNA-1021 was transfected into the LNCaP, DU145 and PC-3 cells, with negative siRNA used as the control. Twenty-four hours after transfection of CtBP2-siRNA-1021, CtBP2 expression was detected by qRT-PCR and the Wes system. Except for the Wes system of CtBP2 in the LNCaP cells which did not achieve a significant difference, RT-PCR and the Wes system results in the other cell lines demonstrated a obvious decrease in CtBP2 compared to the NC group (Fig. 3A and B). Meanwhile, overexpression of CtBP2 in the LNCaP, DU145 and PC-3 cells with the expression plasmid pcDNA3.1 showed opposite results. Except for CtBP2 in the LNCaP cells as detected by the Wes system, RT-PCR and Wes system results in other cell lines demonstrated a notable increase in CtBP2 compared to the NC group (Fig. 3C and D).

Expression levels of CtBP2, FSHR, VEGFA, FHL2 and $S M A D 3$ in PCa cells. qPCR was used to detect the expression of CtBP2, FSHR, VEGFA, FHL2 and SMAD3 after transfection of CtBP2-siRNA-1021 into the LNCaP, DU145 and PC-3 cells (Table II). In the LNCaP cells, FSHR, VEGFA and FHL2 mRNA levels were significantly decreased in the CtBP2-siRNA group when compared with levels in the NC group $(\mathrm{p}<0.001)$. In the DU145 cells, FSHR, VEGFA and SMAD3 showed a significant decrease between the CtBP2siRNA group and the NC group $(\mathrm{p}<0.05)$. In the PC-3 cells, VEGFA and FHL2 showed an obviously significant difference $(\mathrm{p} \leq 0.001)$ (Fig. 4A).

After pcDNA3.1-CtBP2 transfection, FSHR and VEGFA mRNA levels were significantly increased in the LNCaP cells 
A

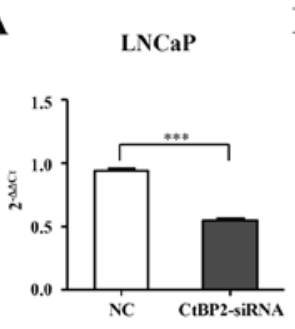

DU145

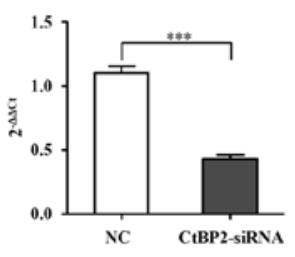

PC3

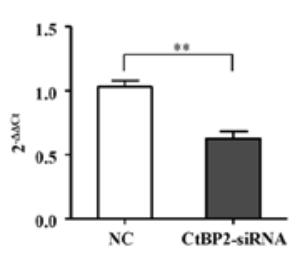

B

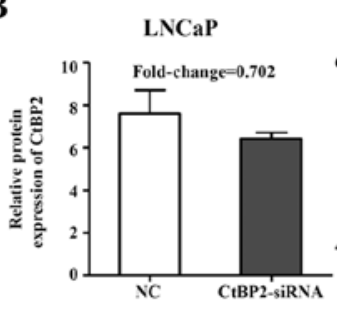

DU145

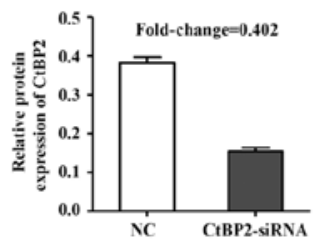

PC3

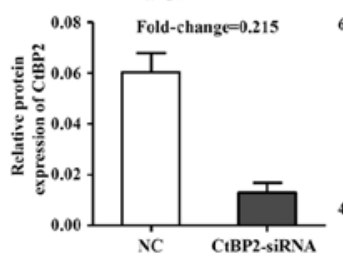

C
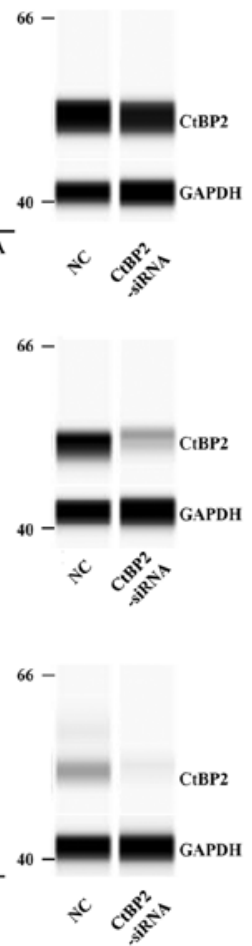

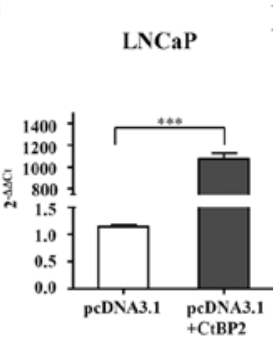

DU145

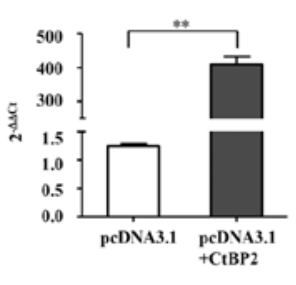

PC3

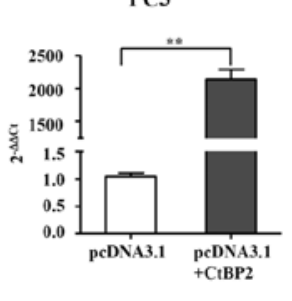

D

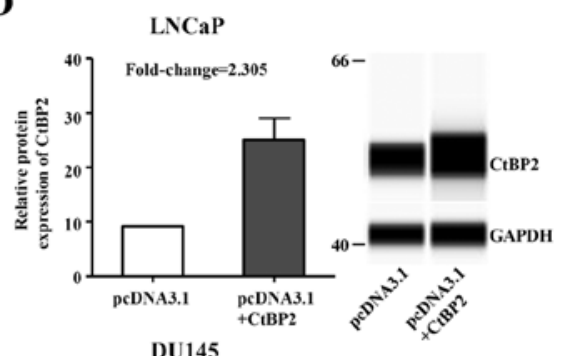

DU145
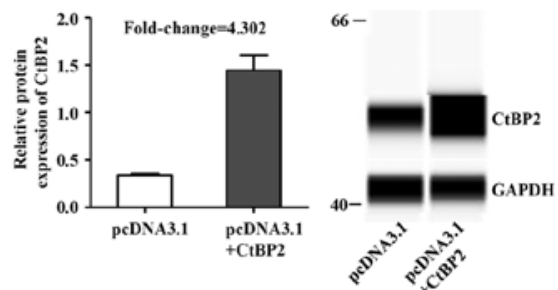

PC3

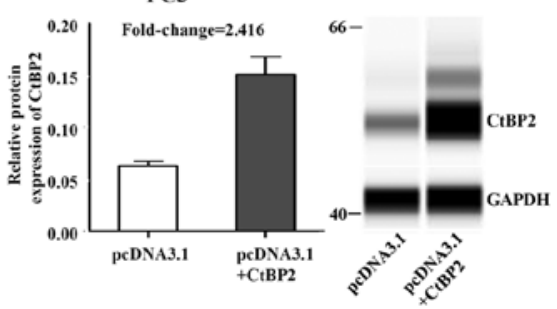

Figure 3. CtBP2 expression in LNCaP, DU145 and PC-3 cells after knockdown and overexpression. (A) LNCaP, DU145 and PC3 cells were transfected with CtBP2-siRNA-1021 and negative siRNA as control. CtBP2 mRNA expression was confirmed by RT-PCR. (B) Knockdown of CtBP2 protein expression was detected by Wes system in the LNCaP, DU145 and PC3 cells. GAPDH was included as a normalization control. (C) LNCaP, DU145 and PC3 cells were transfected with pcDNA3.1 containing CtBP2 and empty vector as control. RT-PCR was used to detect CtBP2 mRNA expression. (D) Overexpression of CtBP2 protein was confirmed by Wes system in the LNCaP, DU145 and PC 3 cells. The data are expressed as mean $\pm \mathrm{SEM} .{ }^{*} \mathrm{P}<0.05,{ }^{* *} \mathrm{P}<0.01,{ }^{* * *} \mathrm{P}<0.001$. NC, group transfected with negative siRNA; CtBP2-siRNA, group transfected with CtBP2-siRNA-1021; pcDNA3.1, group transfected with empty vector; pcDNA3.1+CtBP2, group transfected with pcDNA3.1 containing CtBP2. CtBP, C-terminal binding protein.

A

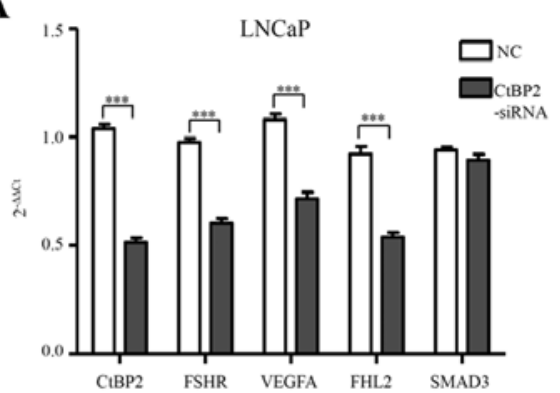

B

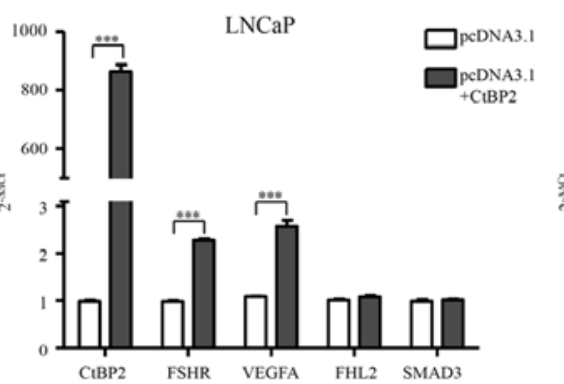

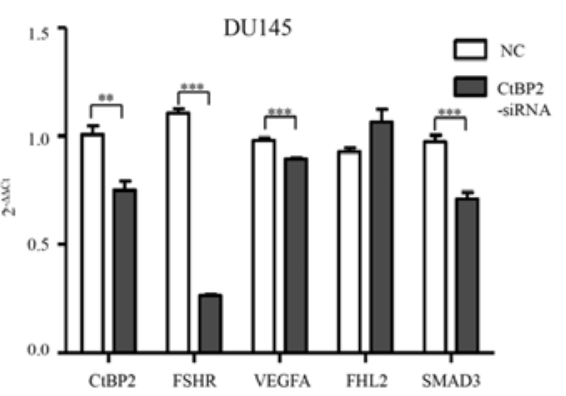
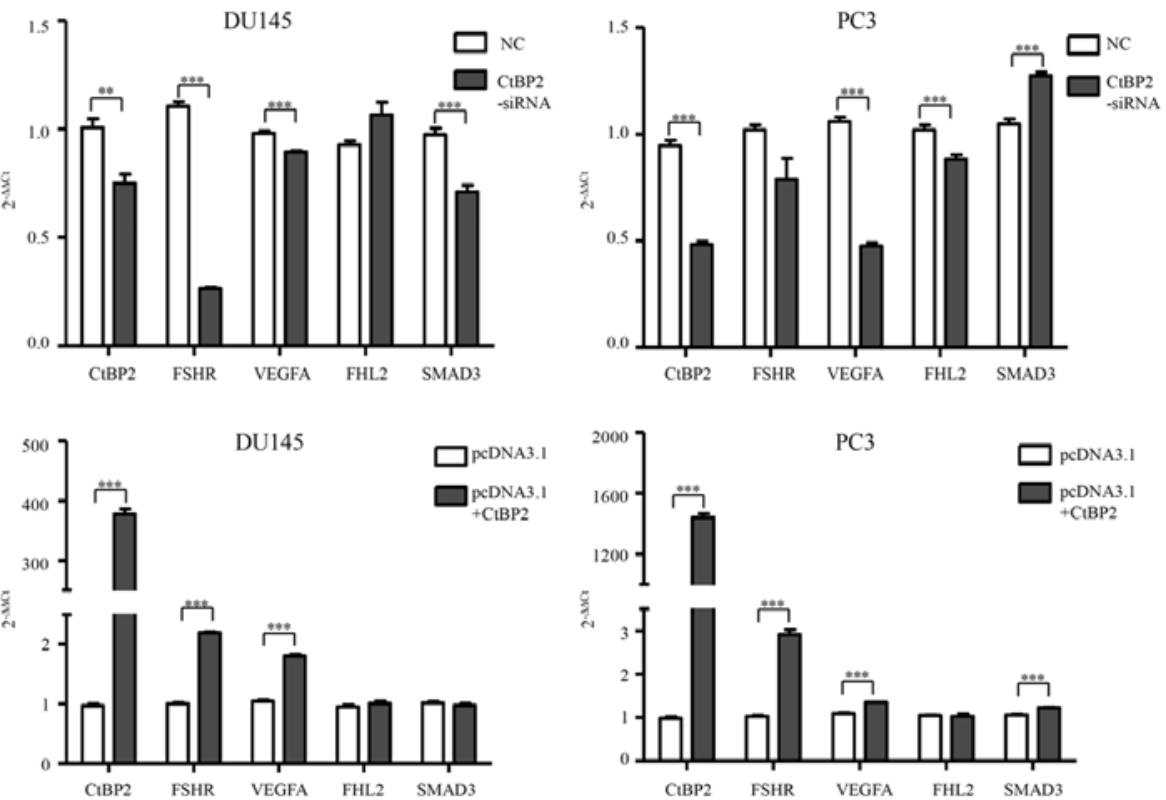

Figure 4. Expression levels of CtBP2, FSHR, VEGFA, FHL2 and SMAD3 in PCa cells. (A) qPCR was used to detect the expression of CtBP2, FSHR, VEGFA, FHL2 and SMAD3 after transfection with CtBP2-siRNA-1021 and negative siRNA in the LNCaP, DU145 and PC3 cells. (B) qPCR was used to detect the expression of CtBP2, FSHR, VEGFA, FHL2 and SMAD3 after transfection with pcDNA3.1 containing CtBP2 and empty vector in LNCaP, DU145 and PC3 cells. Data are expressed as mean $\pm \mathrm{SEM} .{ }^{*} \mathrm{P}<0.05,{ }^{* *} \mathrm{P}<0.01,{ }^{* * *} \mathrm{P}<0.001$. NC, group transfected with negative siRNA; CtBP2-siRNA, group transfected with CtBP2-siRNA-1021; pcDNA3.1, group transfected with empty vector; pcDNA3.1+CtBP2, group transfected with pcDNA3.1 containing CtBP, C-terminal binding protein; FSHR, follicle stimulating hormone receptor; VEGF, vascular endothelial growth factor; FHL2, four and a half LIM domains 2; SMAD3, SMAD family member 3 . PCa, prostate cancer. 
A

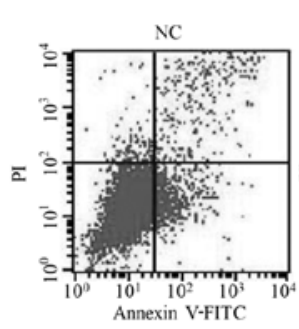

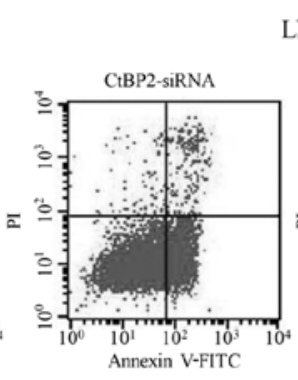

$\mathrm{LNCaP}$

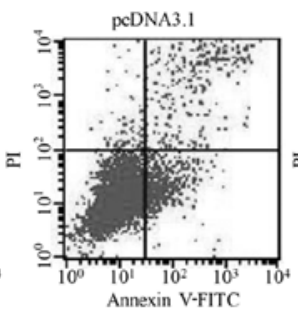

DU145
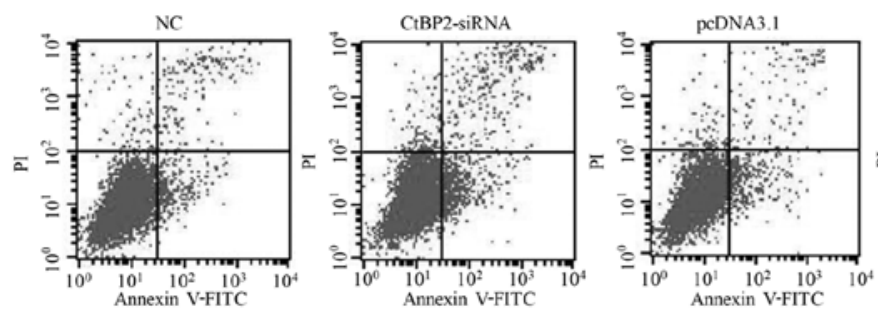

PC3
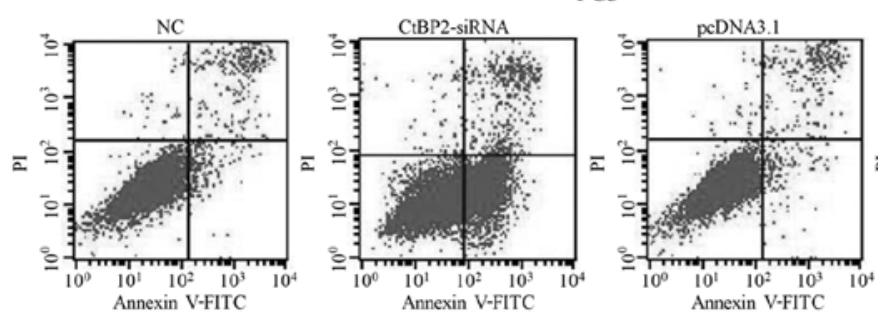
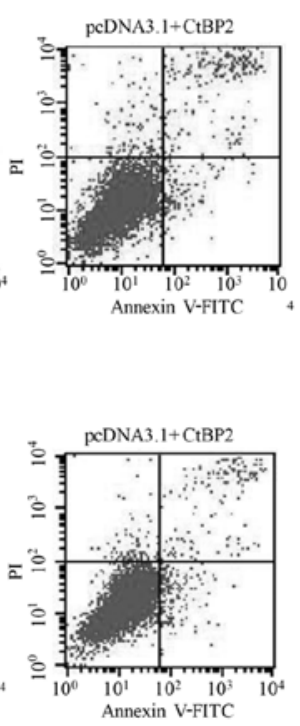

B
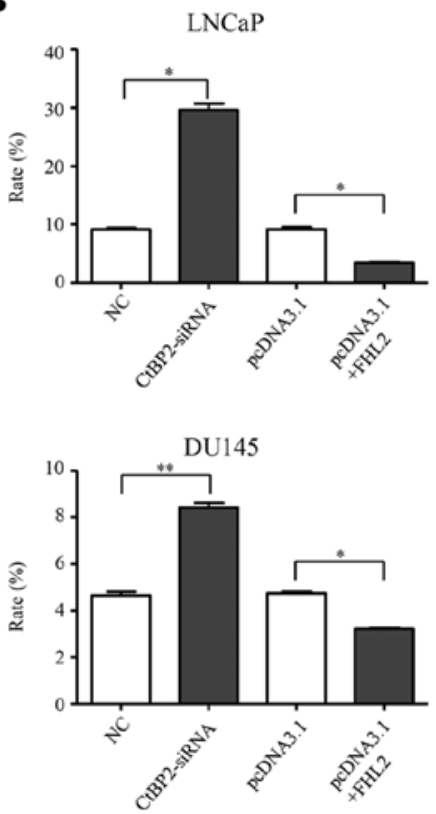

PC3
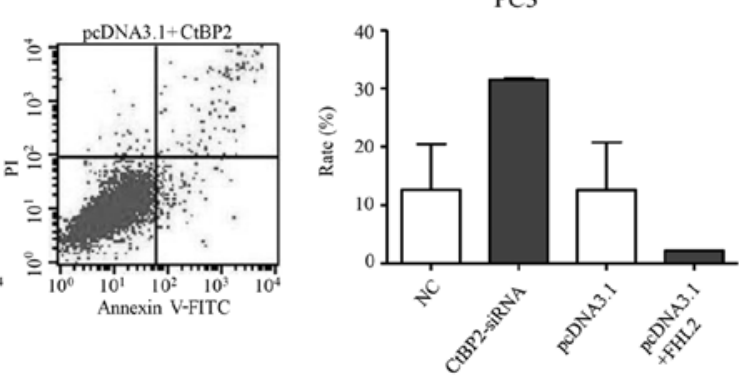

Figure 5. Cell apoptosis of LNCaP, DU145 and PC-3 cells. (A) The ratio of apoptosis after transfection with CtBP2-siRNA-1021 or pcDNA3.1 containing CtBP2 in LNCaP, DU145 and PC3 cells. (B) Comparison of the ratio of apoptosis after transfection with CtBP2-siRNA-1021 or pcDNA3.1 containing CtBP2 in LNCaP, DU145 and PC 3 cells. Data are expressed as the mean \pm SEM. ${ }^{*} \mathrm{P}<0.05,{ }^{* *} \mathrm{P}<0.01,{ }^{* * * *} \mathrm{P}<0.001$. NC, group transfected with negative siRNA; CtBP2siRNA, group transfected with CtBP2-siRNA-1021; pcDNA3.1, group transfected with empty vector; pcDNA3.1+CtBP2, group transfected with pcDNA3.1 containing CtBP2. CtBP, C-terminal binding protein.

compared to levels in the pcDNA3.1+CtBP2 and pcDNA3.1 groups $(\mathrm{p}<0.001)$. A significant difference in FSHR and VEGFA mRNA expression was noted in the DU145 cells $(\mathrm{p}<0.001)$. FSHR, VEGFA and SMAD3 mRNA expression also showed a significant difference $(\mathrm{p}<0.001)$ in the PC-3 cells (Fig. 4B).

Taken together, interference with the expression of CtBP2 effectively affected the expression of FSHR and VEGFA. This indicates that CtBP2 may play an important role in angiogenesis.

Cell apoptosis of LNCaP, DU145 and PC-3 cells. Forty-eight hours after transfection, flow cytometry was used to determine the cell apoptotic rate. CtBP2-siRNA-1021 promoted cell apoptosis in the LNCaP, DU145 and PC-3 cells (Fig. 5A). There was a significant increased in the cell apoptosis rate in the CtBP2-siRNA group compared to that in the NC group in the LNCaP and DU145 cells ( $\mathrm{t}=18.377, \mathrm{p}=0.024$ and $t=15.080, p=0.006$, respectively). However, there was no significant difference in the cell apoptosis rate between the CtBP2-siRNA group and the NC group in the PC-3 cells $(\mathrm{p}=0.250)$ (Fig. 5B).
CtBP2 overexpression inhibited the apoptosis levels in the LNCaP, DU145 and PC-3 cells $48 \mathrm{~h}$ after transfection (Fig. 5A). There was a significant decreased in the cell apoptosis rate between the pcDNA3.1+CtBP2 group and the pcDNA3.1 group in the LNCaP and DU145 cells $(\mathrm{t}=16.423$, $p=0.035$ and $t=15.080, p=0.006$, respectively). There was also no significant difference between the cell apoptosis rate in the pcDNA3.1+CtBP2 group and the pcDNA3.1 group in the PC-3 cells $(\mathrm{p}=0.427)$ (Fig. 5B).

Expression of downstream signaling markers in LNCaP, DU145 and PC 3 cells. To identify the biological consequences of CtBP2 interference, we next investigated the expression of subsequent downstream markers including IL-8, AT2R, CCND1 and MMP9. After knockdown and overexpression of CtBP2 in LNCaP, DU145 and PC-3 cells, qPCR was used to detect the expression of IL-8, AT2R, CCND1 and MMP9. The primers of CtBP2-associated downstream genes are shown in Table II. IL-8, AT2R, CCND1 and MMP9 were significantly decreased between the CtBP2-siRNA and the NC group in the LNCaP, DU145 and PC-3 cells (Fig. 6A). In regards to pcDNA3.1 containing CtBP2, IL-8, AT2R, CCND1 and 
A

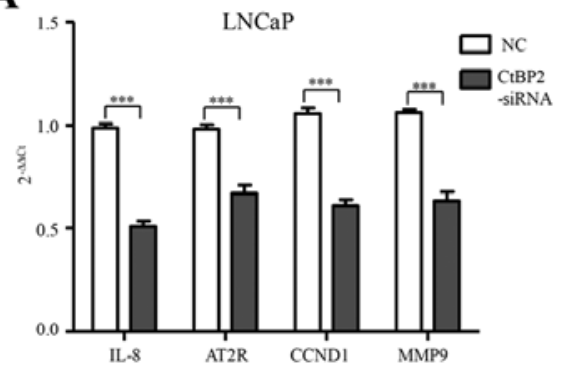

B

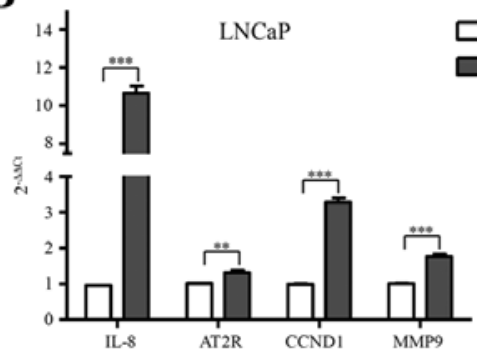

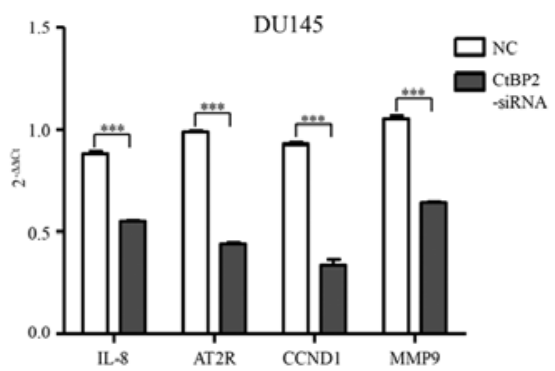
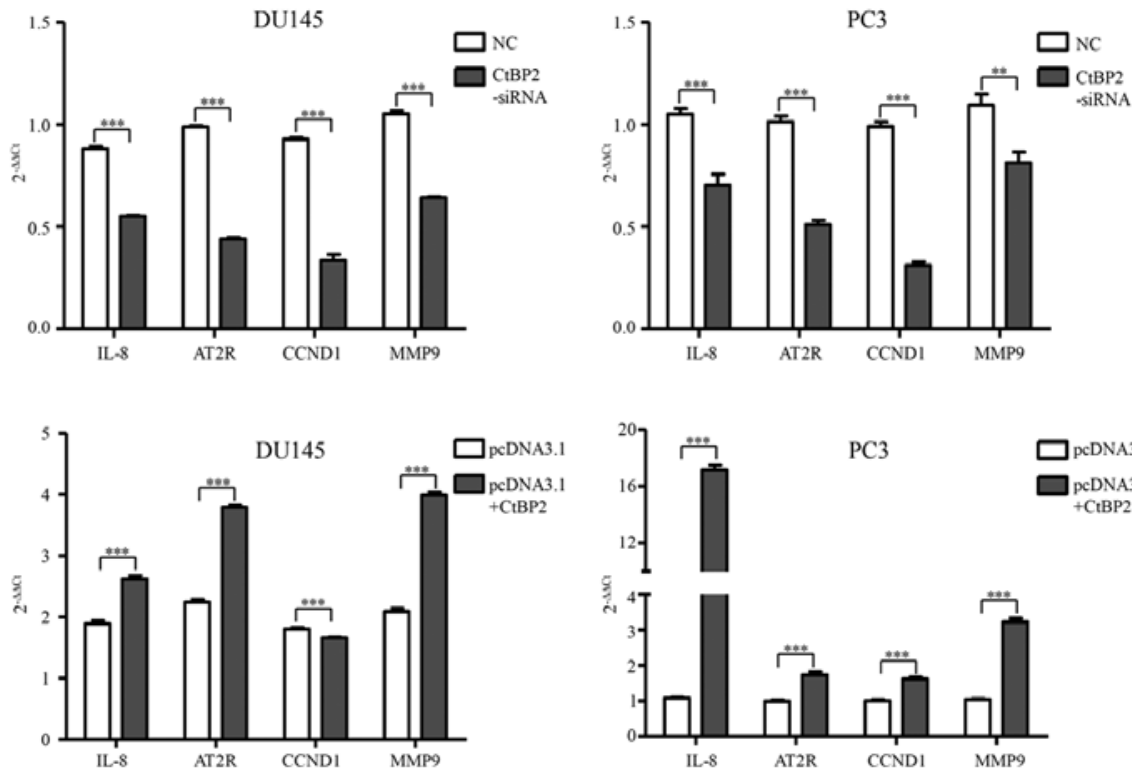

Figure 6. Expression of downstream signaling markers in LNCaP, DU145 and PC3 cells. (A) siRNA knockdown of CtBP2 expression inhibited downstream signaling in LNCaP, DU145 and PC3 cells. qPCR was used to detect the expression of IL-8, AT2R, CCND1 and MMP9. (B) CtBP2 overexpression promoted downstream signaling in LNCaP, DU145 and PC3 cells. qPCR was used to detect the expression of IL-8, AT2R, CCND1 and MMP9. Data are expressed as mean \pm SEM. ${ }^{*} \mathrm{P}<0.05,{ }^{* *} \mathrm{P}<0.01,{ }^{* * *} \mathrm{P}<0.001$. NC, group transfected with negative siRNA; CtBP2-siRNA, group transfected with CtBP2-siRNA-1021; pcDNA3.1, group transfected with empty vector; pcDNA3.1+CtBP2, group transfected with pcDNA3.1 containing CtBP2. CtBP, C-terminal binding protein; IL-8, interleukin 8; AT2R, angiotensin II receptor type 2; CCND1, cyclin D1; MMP9, matrix metallopeptidase 9.

MMP9 showed a significant increase in the LNCaP and PC-3 cells compared with the pcDNA3.1 group. Additionally, IL-8, AT2R and MMP9 were significantly increased in the DU145 cells compared with that in the pcDNA3.1 group (Fig. 6B).

\section{Discussion}

CtBP2 is a transcriptional co-repressor which has been observed to mediate the repression of the tumor-suppressor gene product, p16(INK4A) (10). Takayama et al (13) demonstrated that $\mathrm{CtBP} 2$ modulated the androgen receptor to promote PCa cell proliferation through c-Myc signaling and also promotes PCa progression (11). Aberrant expression of CtBP2 has been found to be associated with tumorigenesis, tumor progression and poor prognosis of PCa $(12,26)$. In this study, we provide evidence that $\mathrm{CtBP} 2$ is closely related with angiogenesis in $\mathrm{PCa}$, and its expression change significantly affects the apoptosis of PCa cells (LNCaP, DU145 and PC-3) and the downstream markers IL-8, AT2R and MMP9.

Formation of functional vasculature is an essential requirement for most physiological processes and the growth of mammalian tissues. Angiogenesis, the formation of new blood vessels from pre-existing vasculature, is important for tumor development and progression (27). Our bioinformatic analysis indicated that the top 6 pathways of CtBP2-related genes were correlated with angiogenesis. VEGF and its receptors VEGFR1/VEGFR2 play major roles in controlling angiogenesis, including vascularization of solid tumors. VEGF-induced neoangiogenesis is mediated by NAADP and two-pore channel-2-dependent $\mathrm{Ca}^{2+}$ signaling (27). Arrhythogenic ARVC and VSMC pathways are closely related with the vascular system. The principal mechanisms that regulate the contractile state of VSMCs are changes in the concentration of cytosolic $\mathrm{Ca}^{2+}\left(\left[\mathrm{Ca}^{2+}\right]_{c}\right)(28)$. Axon guidance represents a key stage in the formation of neuronal network. Meucci et al (29) identified that Axon guidance and focal adhesion pathways regulate cell motility, tumor invasion and angiogenesis leading to less aggressive tumors in older age patients. Dilated cardiomyopathy pathway is also reported to be related to angiogenesis (30).

The 5 overlapping genes (CtBP2, FSHR, VEGFA, FHL2 and SMAD3) obtained by bioinformatic analysis showed that there was a significant expression difference in these genes between PCa and normal adjacent tissues. Following knockdown and overexpression of $\mathrm{CtBP} 2$, the expression of FSHR and VEGFA were notably affected in all cell lines. In most of the experiments, the expression of FHL2 and SMAD3 was also markedly affected by CtBP2 interference. VEGFA can induce proliferation and migration of vascular endothelial cells, and is essential for both physiological and pathological angiogenesis (31). FSH is as efficatious as VEGF which is a well-characterized angiogenic factor in promoting angiogenic processes, and is a highly selective tumor vasculature marker, which is abundant in both primary and metastatic tumors $(16,17)$. FHL2 serves a repressor function in cardiomyocytes through its ability to inhibit ERK1/2 transcriptional coupling (23). SMAD3 is essential for signaling of transforming growth factor $\beta$ (TGF- $\beta$ ) pathway. A role for TGF- $\beta$ in modulating VEGF release has been proposed in several cell types, such as glioblastoma (24). These results indicate that $\mathrm{CtBP} 2$ plays an important role in angiogenesis.

The association between CtBP2 overexpression and tumorigenesis and poor clinical outcome and tumor progression of PCa has been reported $(11,12)$. In this study, we further 
demonstrated that the alteration in $\mathrm{CtBP} 2$ expression obviously affected the apoptosis of PCa cells (LNCaP, DU145 and PC3). Zhang et al (25) revealed that suppressed expression of CtBP2 in PCa PC3 cells markedly inhibited cell proliferation by inducing apoptosis in vitro. In this study, we further confirmed that inhibition of $\mathrm{CtBP} 2$ induced apoptosis in $\mathrm{PCa}$ cells (LNCaP, DU145 and PC3). On the contrary, upregulation of CtBP2 inhibited the apoptosis of PCa cells. A more recent study demonstrated a critical function for CtBPs in the transcriptional repression of pro-apoptotic genes such as Bax, Puma, Bik, and Noxa (32).

In conclusion, $\mathrm{CtBP} 2$ expression is associated with $\mathrm{PCa}$ development and progression. We integrated and analyzed 4 datasets from CtBP2 and FSHR GWAS, CtBP2 binding data and the significant difference in PCa mRNA from the TCGA database, and performed pathway enrichment. We revealed that the top 6 pathways were closely related with angiogenesis. CtBP2 interference indicated that its expression alteration also affected the expression of VEGFA, FSHR, FHL2 and SMAD3 which are closely related with angiogenesis. In addition, CtBP2 markedly affected the apoptosis of $\mathrm{PCa}$ cells in vitro, and impacted the expression of IL-8, AT2R, CCND1 and MMP9 which are associated with cancer progression. These results highlight the association between CtBP2 and angiogenesis in $\mathrm{PCa}$ and indicate that $\mathrm{CtBP} 2$ may be a potential therapeutic target for $\mathrm{PCa}$. The molecular mechanisms underlying the regulation of angiogenesis by $\mathrm{CtBP} 2$ warrant further in vitro and in vivo investigation.

\section{Acknowledgements}

This research was supported by grants from the Anhui Natural Science Foundation (no. 1408085MH181), Anhui Educational Departmental Function (KJ2012 Z181), National Natural Science Foundation of China (nos. 81272853 and 81472414), and the Guangxi Natural Science Foundation (nos. 2015GXNSFBB139008 and 2014GXNSFBA118201).

\section{References}

1. Torre LA, Bray F, Siegel RL, Ferlay J, Lortet-Tieulent J and Jemal A: Global cancer statistics, 2012. CA Cancer J Clin 65: 87-108, 2015.

2. DiBlasio CJ, Malcolm JB, Hammett J, Wan JY, Aleman MA, Patterson AL, Wake RW and Derweesh IH: Survival outcomes in men receiving androgen-deprivation therapy as primary or salvage treatment for localized or advanced prostate cancer: 20-year single-centre experience. BJU Int 104: 1208-1214, 2009.

3. Ramsay AK, McCracken SR, Soofi M, Fleming J, Yu AX, Ahmad I, Morland R, Machesky L, Nixon C, Edwards DR, et al: ERK5 signalling in prostate cancer promotes an invasive phenotype. Br J Cancer 104: 664-672, 2011.

4. Maruyama Y, Miyazaki T, Ikeda K, Okumura T, Sato W, Horie-Inoue K, Okamoto K, Takeda S and Inoue S: Short hairpin RNA library-based functional screening identified ribosomal protein L31 that modulates prostate cancer cell growth via p53 pathway. PLoS One 9: e108743, 2014.

5. Turner J and Crossley M: The CtBP family: Enigmatic and enzymatic transcriptional co-repressors. BioEssays 23: 683-690, 2001.

6. Chinnadurai G: CtBP, an unconventional transcriptional corepressor in development and oncogenesis. Mol Cell 9: 213-224, 2002.

7. May T, Yang J, Shoni M, Liu S, He H, Gali R, Ng SK, Crum C, Berkowitz RS and Ng SW: BRCA1 expression is epigenetically repressed in sporadic ovarian cancer cells by overexpression of C-terminal binding protein 2. Neoplasia 15: 600-608, 2013.
8. Deng H, Liu J, Deng Y, Han G, Shellman YG, Robinson SE, Tentler JJ, Robinson WA, Norris DA, Wang XJ, et al: CtBP1 is expressed in melanoma and represses the transcription of pl6INK4a and Brcal. J Invest Dermatol 133: 1294-1301, 2013.

9. Di LJ, Byun JS, Wong MM, Wakano C, Taylor T, Bilke S, Baek S, Hunter K, Yang H, Lee M, et al: Genome-wide profiles of CtBP link metabolism with genome stability and epithelial reprogramming in breast cancer. Nat Commun 4: 1449, 2013

10. Guan C, Shi H, Wang H, Zhang J, Ni W, Chen B, Hou S, Yang X, Shen A and Ni R: CtBP2 contributes to malignant development of human esophageal squamous cell carcinoma by regulation of p16 ${ }^{\mathrm{INK} 4 \mathrm{~A}}$. J Cell Biochem 114: 1343-1354, 2013.

11. Debiais-Delpech C, Godet J, Pedretti N, Bernard FX, Irani J, Cathelineau X, Cussenot O and Fromont G: Expression patterns of candidate susceptibility genes HNF1 $\beta$ and $\mathrm{CtBP} 2$ in prostate cancer: Association with tumor progression. Urol Oncol 32: 426-432, 2014.

12. Zhang C, Li S, Qiao B, Yang K, Liu R, Ma B, Liu Y, Zhang Z and $\mathrm{Xu}$ Y: CtBP2 overexpression is associated with tumorigenesis and poor clinical outcome of prostate cancer. Arch Med Sci 11: 1318-1323, 2015.

13. Takayama K, Suzuki T, Fujimura T, Urano T, Takahashi S, Homma Y and Inoue S: CtBP2 modulates the androgen receptor to promote prostate cancer progression. Cancer Res 74: 6542-6553, 2014.

14. Ferrara N and Kerbel RS: Angiogenesis as a therapeutic target. Nature 438: 967-974, 2005.

15. Roukens MG, Alloul-Ramdhani M, Baan B, Kobayashi K, Peterson-Maduro J, van Dam H, Schulte-Merker S and Baker DA: Control of endothelial sprouting by a Tel-CtBP complex. Nat Cell Biol 12: 933-942, 2010.

16. Stilley JA, Guan R, Duffy DM and Segaloff DL: Signaling through FSH receptors on human umbilical vein endothelial cells promotes angiogenesis. J Clin Endocrinol Metab 99: E813-E820, 2014.

17. Yang D, Feng L, Dougherty CA, Luker KE, Chen D, Cauble MA, Banaszak Holl MM, Luker GD, Ross BD, Liu Z, et al: In vivo targeting of metastatic breast cancer via tumor vasculature-specific nano-graphene oxide. Biomaterials 104: 361-371, 2016.

18. Qin B, Zhou M, Ge Y, Taing L, Liu T, Wang Q, Wang S, Chen J, Shen L, Duan X, et al: CistromeMap: A knowledgebase and web server for ChIP-Seq and DNase-Seq studies in mouse and human. Bioinformatics 28: 1411-1412, 2012.

19. Stranger BE, Forrest MS, Dunning M, Ingle CE, Beazley C, Thorne N, Redon R, Bird CP, de Grassi A, Lee C, et al: Relative impact of nucleotide and copy number variation on gene expression phenotypes. Science 315: 848-853, 2007.

20. Wang P, Dai M, Xuan W, McEachin RC, Jackson AU, Scott LJ, Athey B, Watson SJ and Meng F: SNP Function Portal: A web database for exploring the function implication of SNP alleles. Bioinformatics 22: e523-e529, 2006.

21. Dennis G Jr, Sherman BT, Hosack DA, Yang J, Gao W, Lane HC and Lempicki RA: DAVID: Database for Annotation, Visualization, and Integrated Discovery. Genome Biol 4: 3, 2003.

22. Joyal JS, Sun Y, Gantner ML, Shao Z, Evans LP, Saba N, Fredrick T, Burnim S, Kim JS, Patel G, et al: Corrigendum: Retinal lipid and glucose metabolism dictates angiogenesis through the lipid sensor Ffarl. Nat Med 22: 692, 2016.

23. Purcell NH, Darwis D, Bueno OF, Müller JM, Schüle R and Molkentin JD: Extracellular signal-regulated kinase 2 interacts with and is negatively regulated by the LIM-only protein FHL2 in cardiomyocytes. Mol Cell Biol 24: 1081-1095, 2004.

24. Seystahl K, Tritschler I, Szabo E, Tabatabai G and Weller M: Differential regulation of TGF- $\beta$-induced, ALK-5-mediated VEGF release by SMAD2/3 versus SMAD1/5/8 signaling in glioblastoma. Neuro Oncol 17: 254-265, 2015.

25. Zhang C, Gao C, Xu Y and Zhang Z: CtBP2 could promote prostate cancer cell proliferation through c-Myc signaling. Gene 546: 73-79, 2014.

26. Chung AS and Ferrara N: Developmental and pathological angiogenesis. Annu Rev Cell Dev Biol 27: 563-584, 2011.

27. Favia A, Desideri M, Gambara G, D'Alessio A, Ruas M, Esposito B, Del Bufalo D, Parrington J, Ziparo E, Palombi F, et al: VEGF-induced neoangiogenesis is mediated by NAADP and two-pore channel-2-dependent $\mathrm{Ca}^{2+}$ signaling. Proc Natl Acad Sci USA 111: E4706-E4715, 2014. 
28. Akata T: Cellular and molecular mechanisms regulating vascular tone. Part 2: Regulatory mechanisms modulating $\mathrm{Ca}^{2+}$ mobilization and/or myofilament $\mathrm{Ca}^{2+}$ sensitivity in vascular smooth muscle cells. J Anesth 21: 232-242, 2007.

29. Meucci S, Keilholz U, Tinhofer I and Ebner OA: Mutational load and mutational patterns in relation to age in head and neck cancer. Oncotarget 7: 69188-69199, 2016.

30. Yue X, Lin X, Yang T, Yang X, Yi X, Jiang X, Li X, Li T, Guo J, Dai Y, et al: Rnd3/RhoE modulates hypoxia-inducible factor $1 \alpha /$ vascular endothelial growth factor signaling by stabilizing hypoxia-inducible factor $1 \alpha$ and regulates responsive cardiac angiogenesis. Hypertension 67: 597-605, 2016.
31. Aryal B, Shimizu T, Kadono J, Furoi A, Komokata T, Inoue M, Ikeda S, Fukukura Y, Nakamura M, Yamakuchi M, et al: A switch in the dynamics of intra-platelet VEGF-A from cancer to the later phase of liver regeneration after partial hepatectomy in humans. PLoS One 11: e0150446, 2016.

32. Stankiewicz TR, Gray JJ, Winter AN and Linseman DA C-terminal binding proteins: Central players in development and disease. Biomol Concepts 5: 489-511, 2014. 